\title{
Flame temperature and alkali emission in combustion of sugarcane bagasse and straw
}

\author{
Carlos T. Salinas ${ }^{1,2 *}$, Leonardo Gonzales ${ }^{1}$, Luis Felipe Bellido ${ }^{1}$, Lucas Ramos $^{1}$, Daniel Marcelo-Aldana ${ }^{1}$ \\ ${ }^{1}$ University of Piura, Ramón Mugica 131, Av., Urb. San Eduardo, Piura, Peru \\ ${ }^{2}$ Santa Cecilia University, Postgraduate Program of Mechanical Engineering, Rua Cesário Mota 08, Sala F-34, Santos, Brazil
}

\begin{abstract}
This work presents a numerical and experimental study in a laboratorial rig to measure local flame temperature and alkali emission during the combustion of sugarcane bagasse and straw. Tubular combustion chamber to simulate combustion in grate is used. A spectral analysis method in the visible spectrum was used for measurement of local temperatures in a sugarcane bagasse flame and sugarcane straw flame. The two-color method is used to calculate flame temperature. In flame reactive zone, the bagasse and straw flame temperatures in the range of 1420 to $1510 \mathrm{~K}$ and in the range of 1450 to $1550 \mathrm{~K}$ respectively are calculated. The intensity of alkali emission during the sugarcane bagasse and straw combustion is calculated using data of the spectrum in visible range. It was found that potassium emission is correlated with the instantaneous bagasse flame temperature in the reactive combustion zone. Emission of potassium and sodium during straw combustion is higher than in bagasse combustion. Temperature and alkali emissions along the flame height are measured.
\end{abstract}

\section{Introduction}

In recent years, the combustion behaviour of sugarcane bagasse against different types of fossil fuels has been investigated. Sugarcane bagasse is the residual biomass from the sugarcane milling.

In the study of combustion systems, temperature and radiative properties are important parameters. The knowledge of the real temperature is essential for the optimization of combustion systems [1]. Combustion products are mainly composed of triatomic gases and solid particles, such as carbon dioxide, water vapor, and soot. The radiative properties of triatomic gases show strong spectral selectivity, and the characteristics of soot particles are closely related to temperature, wavelength and radiation. Radiation thermometry based on colour image processing has proven to be effective in measuring flame temperature and allows cost savings [2]. Colour CCD cameras were used to investigate the simultaneous measurement of temperature distribution and radiative properties by radiation analysis [3]. From measurements of the temperature distribution in a candle flame, it was determined that the behaviour of emissivity is approximately inverse to that of wavelength [4]. To improve the accuracy of the temperature calculation, a multi-wavelength technique was proposed to introduce an emissivity ratio model to replace the grey body assumption. This method showed more accurate results compared to the traditional twocolour method [5].

The sugarcane bagasse in many cases is rich in potassium, released during the combustion generating corrosion and incrustation in the heat exchangers that reducing their efficiency. The information about the correlation between temperature and concentration of alkaline gases allows to optimize the operation of thermoelectric boilers that use solids wastes as fuel, improving their efficiency and reducing maintenance costs $[6,7]$. For studies in industrial furnaces, flame emission spectroscopy (FES) is a simple and less expensive method, as it does not depend on devices such as lasers. The potassium and chlorine released in the combustion of sugarcane residues, originating in a large part of $\mathrm{KCl}$, generate corrosion in the pipes and heat exchangers, as they react with their protective layers based on iron oxide chromium [8]. Studies on the emission of alkali metals in the combustion of municipal solid waste in industrial incinerators, where not only potassium, but also sodium is present, were carried out using flame emission spectroscopy (FES) $[9,10]$.

The use of energy systems that take advantage of solid biomass residues has increased in recent years in Peru. The use of these energy sources from biomass residues originating from industrial processes allows the generation of clean energy. The use of biomass means a reduction in carbon dioxide emissions compared to conventional systems.

The objective of this work was to estimate the temperature and potassium emission in the combustion of sugarcane bagasse using the flame emission spectroscopy (FES) method. The experiments were carried out in a grid-type pilot combustion chamber set up in a Biomass Combustion Laboratory at University of Piura, Perú.

\section{Experimental rig and numerical method}

\subsection{Experimental rig}

The biomass combustion process depends on many factors, such as the composition of the fuel, the geometry of the particles, the design of the combustion chamber and the air distribution. An experimental test ring is mounted in the laboratory to obtain the spectrum of the bagasse or straw flame during the combustion process to calculate the flame temperature. A vertical tubular combustion chamber designed to simulate bagasse combustion in grate was used. A scheme of combustion chamber is show in Figure 1. Bagasse is composed of fiber and water-soluble materials, mainly sugar and impurities. The bagasse samples for the combustion tests at laboratory were collected at a power plant in Piura region, in the coast north of Peru. The 
weather in this region is hot, and reports of power plant indicate bagasse moisture in the range of $10 \%$.

The spectrometer system used to perform the in tests with bagasse and straw consists of two spectrometers, a measuring probe, and a portable computer. An AvaSpec-ULS2048XL-RS-EVO and an AvaSpecULS4096CL-RS-EVO Fiber-Optic Spectrometers were used to process the incoming light data. The measurement wavelength range of the spectrometers were $200 \mathrm{~nm}$ to $720 \mathrm{~nm}$ and $600 \mathrm{~nm}$ to $1100 \mathrm{~nm}$ respectively. The spectral resolution of the spectrometers are $0.6 \mathrm{~nm}$ and $0.13 \mathrm{~nm}$ respectively. The measuring probe consists of a collimating lens and a fiber-optic cable. The COL-UV/vis collimating lens, screws onto the end of the fiber optic entrance connector and converts the divergent beam of radiation into a parallel beam. The spectrometer is connected to a portable computer through a USB cable via and AvaSoft-8 USB2 interface.
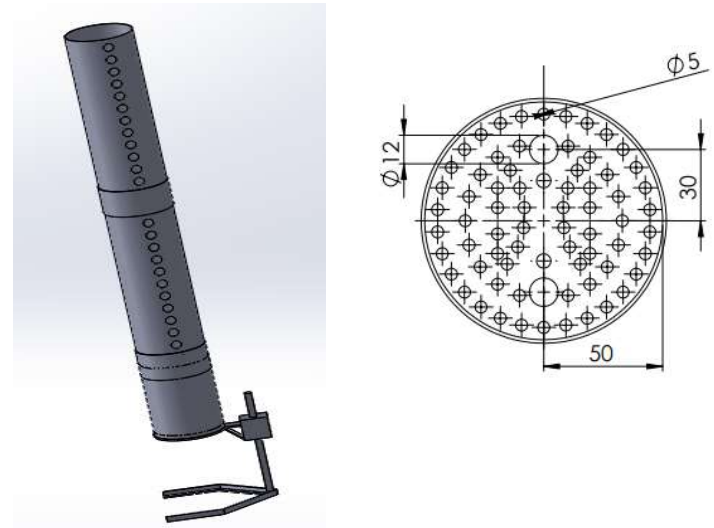

Fig. 1. (a) Scheme of combustion chamber (b) Grate geometry.

The combustion process is carried out in a batch procedure using samples of $10 \mathrm{~g}$ of sugarcane bagasse or straw. An LPG Bunsen burner is used to sustain the biomass combustion. For experiments reported in this paper, the exposure (integration) time is about several hundred milliseconds, which means the measurement of radiative intensifies at different wavelengths by the spectrometer is performed almost simultaneously. The spectrometric system was used to collect spectral intensities of sugarcane bagasse flame through the viewing port at chamber every one second.

\subsection{Numerical method}

Planck's law for black bodies, idealized bodies that, for any temperature, emit maximum of radiation at each wavelength of the spectrum in a diffuse way (regardless of direction) and that absorb all incident radiation, can be adapted with inclusion of emissivity $(\varepsilon)$ to relate the spectral emissive power to the temperature of real bodies as (1) [11].

$$
E(\lambda, T)=\varepsilon \frac{2 \pi h c^{2}}{\lambda^{5}\left(e^{\left.\frac{C_{2}}{\lambda T}-1\right)}\right.} \quad\left(W / m^{2} n m\right)
$$

where $\lambda$ is the wavelength for which the spectral emissive power is being determined $(E)$, T is the body temperature, h Planck's constant, $c$ is the speed of light and $C_{2}$ is $h c / k$ where $k$ is the Boltzmann constant [11]. The temperature equation for the two-color method is obtained by selecting two wavelengths, $\lambda_{i}$ and $\lambda_{j}$, of a spectrum collected by the spectrometer with the body at temperature $T$. Dividing $E\left(\lambda_{i}, T\right) / E\left(\lambda_{j}, T\right)$ on both sides of (1) and isolating the $T$ we get the temperature equation of the two-color method (2). As the spectrometer provides the emissive power for different wavelengths (spectral), it is possible to estimate the temperature several times by varying the wavelength pairs [12].

$$
T=\frac{C_{2}\left(1 / \lambda_{j}{ }^{-1 / \lambda_{i}}\right)}{\left(\operatorname{Ln}\left(E\left(\lambda_{i}, T\right) / E\left(\lambda_{j}, T\right)\right)+\operatorname{Ln}\left({ }^{\varepsilon_{j}} / \varepsilon_{i}\right)+5 \operatorname{Ln}\left(\lambda_{i} / \lambda_{j}\right)\right)}
$$

where $T$ is the estimated temperature. $\varepsilon_{i}$ and $\varepsilon_{j}$ are emissivities at wavelengths $\lambda_{i}$ and $\lambda_{j}$. Soot particles in the flame have a gray body property, a body in which the rate of change in emissivity as a function of variation in wavelengths approaches zero. For nearby $\lambda_{i}$ and $\lambda_{j}$ (less then $128 \mathrm{~nm}$ ), one can simplify (2) if $\varepsilon_{i} / \varepsilon_{j} \cong 1$, which allows us to estimate temperatures using (3) [13]. In step 1 of the work, we used the $30 \mathrm{~nm}$ distance between wavelengths and (3).

$$
T_{i, j}=\frac{C_{2}\left(1 / \lambda_{j}{ }^{-1} / \lambda_{i}\right)}{\left(\operatorname{Ln}\left(I_{i} / I_{j}\right)+5 \operatorname{Ln}\left(\lambda_{i} / \lambda_{j}\right)\right)}
$$

Temperatures are calculated using the two-color method for wavelength pairs $\lambda$ and $\lambda+\Delta \lambda$, with $\Delta \lambda$ equal to 30 $\mathrm{nm}$. Spectral emissive power due to alkali emissions are subtracted in spectral data before temperature calculation.

\section{Results and discussion}

Spectral data of flame emission were collected at every one second during combustion tests for twelve bagasse and straw samples. Figure 2 shows the temperatures calculated using the equation (3) during four experiments of bagasse combustion. Twenty-four spectral data in every experiment were collected. The two-colour method for wavelength pairs $\lambda$ and $\lambda+\Delta \lambda$, with $\Delta \lambda$ equal to $30 \mathrm{~nm}$ was used. The choice of $\Delta \lambda=30 \mathrm{~nm}$ due to the greater stability of the estimated temperatures compared to lower values of $\Delta \lambda$. Temperatures were estimated for the range of wavelengths between $400 \mathrm{~nm}$ to $1000 \mathrm{~nm}$ provided by the spectrometers. The lens is focusing the reactive zone. Flame temperature varies between $1400 \mathrm{~K}$ to 1510 $\mathrm{K}$ along the combustion of every sample o bagasse. Other 8 experiments shown the same flame temperature range. Line of emission at $589.3 \mathrm{~nm}$ corresponding to sodium emission and lines of emission at $766.4 \mathrm{~nm}$ and $769.8 \mathrm{~nm}$ corresponding to potassium emission were detected. Sodium emission is not usually detected in bagasse samples in other studies in the literature. Bottom part of figure 2 shows the curves of the variation 
of the radiation intensity corresponding to the potassium emission in the line of emission line at $766.4 \mathrm{~nm}$. It seems that higher flame temperatures produce higher radiation intensity in potassium emission. The radiation intensity is related to content of potassium emission.

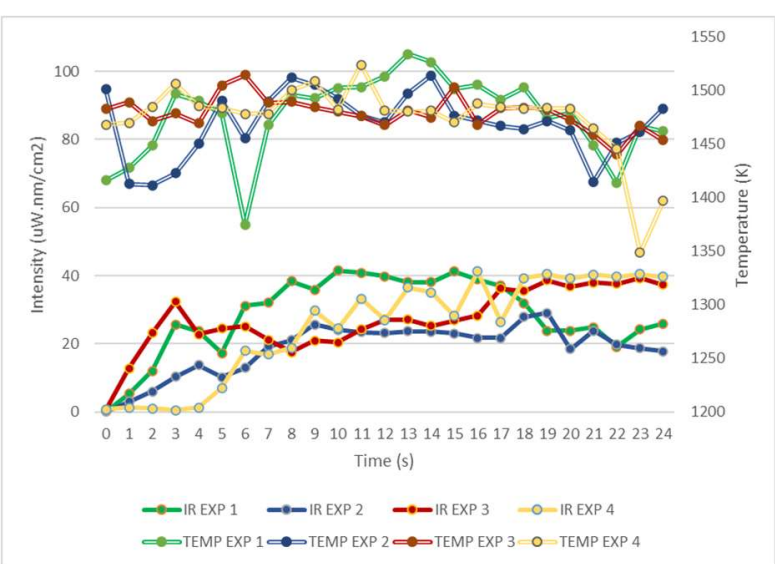

Fig. 2. Temperature and radiation intensity of potassium emission $(766.4 \mathrm{~nm})$ during bagasse samples combustion

In Figure 3 is shown the comparison of radiation intensity for sodium and potassium emission. Potassium emission is higher than sodium emission for the bagasse samples in this study. Potassium emission at $766.4 \mathrm{~nm}$ (intensity K1) slightly higher than potassium emission at $769.8 \mathrm{~nm}$ (intensity K2) was found. Higher temperature and alkali emission at middle of combustion period was found.

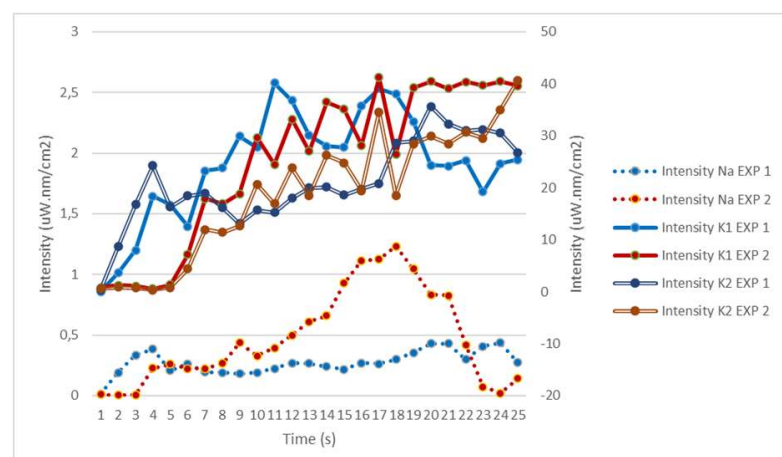

Fig. 3. Comparison of radiation intensity for sodium and potassium emission during bagasse combustion.

Figure 4 shown the variation of temperature and radiation intensity of potassium emission (766.4 nm) during straw combustion. Straw samples were dry. Flame temperatures in the range of $1450 \mathrm{~K}$ to $1540 \mathrm{~K}$ were measured and are slightly higher than flame temperatures during bagasse combustion tests. The period of combustion is less than bagasse combustion for the same sample weight. Based in radiative intensity detection, it can be observed higher potassium emission in straw combustion compared with the emission in bagasse combustion. Similar results is found for potassium emission at $769.8 \mathrm{~nm}$. The higher potassium emission during straw combustion test were detected in the final period of combustion.

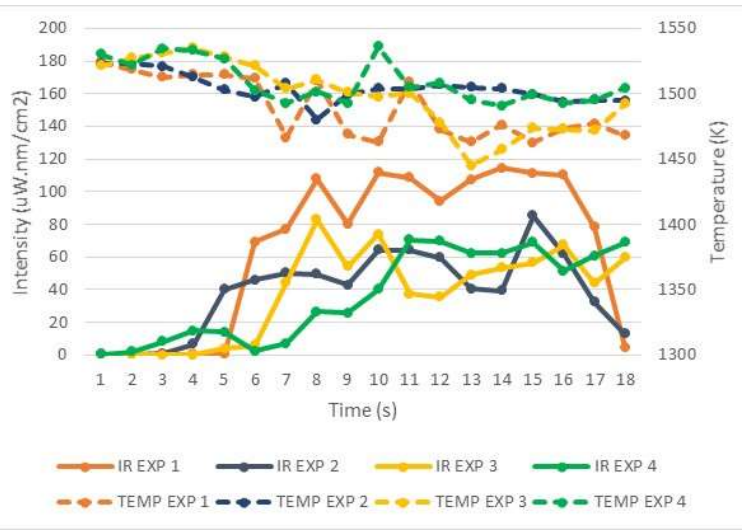

Fig. 4. Variation of temperature and radiation intensity of potassium emission $(766.4 \mathrm{~nm})$ during straw combustion

In Figure 5 is shows the comparison of radiation intensity of sodium emission and potassium emission during a sample straw combustion. Similarly, that bagasse combustion, in this case the potassium emission at $766.4 \mathrm{~nm}$ (intensity K1) and at $669.8 \mathrm{~nm}$ (intensity $\mathrm{K} 2$ ) is higher than sodium emission. Again, potassium emission at $766.4 \mathrm{~nm}$ slightly higher than potassium emission at $769.8 \mathrm{~nm}$ was found. During straw combustion tests, a higher sodium emission in the final period of combustion were detected. Based in radiative intensity emission detection, higher alkali emission, both sodium and potassium during the straw combustion compared with alkali emission during bagasse combustion was found.

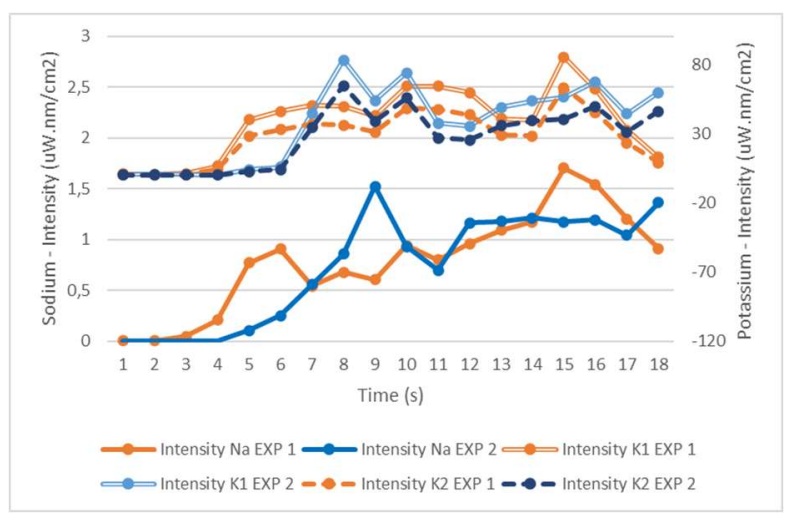

Fig. 5. Comparison of radiation intensity of sodium and potassium emission during a sample straw combustion

\section{Conclusions}

Local flame temperature during combustion of sugarcane bagasse and straw were estimated. Biomass samples come from a new area of sugar cane planting in wastelands on the northern coast of Peru. The twocolour method is used to calculate the flame temperature. The bagasse and straw flame temperatures in the reactive zone of combustion were estimated in the range of 1420 to $1510 \mathrm{~K}$ and 1450 to $1550 \mathrm{~K}$ respectively. For all combustion tests using the same sample weight, the straw flame temperatures were slightly higher than bagasse flame temperatures. Combustion period for straw were less than bagasse 
combustion, among the other, one factor for this was that straw samples were dry. Both, in bagasse combustion and in straw combustion, emission of sodium was detected. Based in detection of radiation intensity of potassium and sodium spectral emission during combustion of bagasse and straw, alkali emission in the combustion of sugarcane bagasse is less than in straw combustion. It seems that higher flame temperatures produce higher radiation intensity in potassium emission. In next works will look to relate alkali radiation intensity to alkali content.

The authors acknowledge the financial support of the Project Concytec (Peru) - World Bank "Improvement and expansion of services of the national system of science, technology and technological innovation" 8682-PE, through its executing unit ProCiencia (Contract 010-2019-Fondecyt).

\section{References}

1. Reza Khatami, Yiannis A. Levendis. On the deduction of single coal particle combustion temperature from three-color optical pyrometry. Combustion and flame, vol 158, 1822 - 1836, 2011.

2. Junyi Lin, Xiangyu Zhang, Kaiyun Liu, Wenjie Zhang. Emissivity characteristics of hydrocarbon flame and temperature measurement by color image Processing. MDPI Energies, vol 12, 2185; doi:10.3390/en12112185, 2019.

3. Lou, C.; Li, W.H.; Zhou, H.C.; Salinas, C.T. (2011); Experimental investigation on simultaneous measurement of temperature distributions and radiative properties in an oil-fired tunnel furnace by radiation analysis. Int. J. Heat Mass Transf, vol 54, 1-8, 2011.

4. Shu Zheng, Huaichun Zhou, Huawei Liu (2019) Measurement of the distribution of temperature and emissivity of a candle flame using hyperspectral imaging technique. Optik vol 183, 222-231,

2019. doi.org/10.1016/j.ijleo.2019.02.077.

5. Licheng Shao, Zhijun Zhou, Liping Chen, Longzhen Guo, Baojia Chen, Lijiang Liang. Study of a improved two-color method integrated with the emissivity ratio model and its application to air and oxy-fuel flame in industrial furnaces. Measurement vol 123, 54-61, 2018.

6. C. Lou, Y. Tian. "Online detection method of concentration in boiler burning". US Patent 9651 48, 2017.

7. V. Barisic, J. Mahanen, E.C. Zabetta. Role of sodium vs. potassium on agglomeration and corrosion in CFB boilers. Impacts of Fuel Quality on Power Production, the 26th International Conference, September 19-23, 2016, Prague, Czech Republic.

8. C.T. Salinas, Y. Pu, C. Lou, D.B. dos Santos. Experiments for combustion temperature measurements in a sugarcane bagasse large-scale boiler furnace. Applied Thermal Engineering, vol. 175, $115433,2020$. doi.org/10.1016/j.applthermaleng.2020.115433

9. X. He, C. Lou, Y. Qiao, M. Lin. In-situ measurement of temperature and alkali metal concentration in municipal solid waste incinerators using flame emission spectroscopy. Waste Management, vol. 102, 486-491, 2020.

10. C. Lou, Y. Pu, Y. Zhao, Y. Bai, B. Yao, D. Yu. An in-situ method for time-resolved sodium release behavior during coal combustion and its application in industrial coal-fired boilers. Proceedings of the Combustion Institute, vol. 38 (3), 4199-4206, 2021.

11. Modest M.F. Radiative heat transfer, 3 ed., Elsevier, 2013.

12. Fu T., Tan P., Pang C., Zhao H., and Shen Y. Fast fiber-optic multi-wavelength pyrometer. Review of Scientific Instruments, vol. 82, 064902, 2011. https://doi: 10.1063/1.3596567.

13. Jan W.J., Lou C., Cheng Q., Zhao P., Zhang X.Y. In situ measurement of alkali metals in a MSW incinerator using spontaneous emission spectrum, Appl. Sciences 7 (3), 263, 2017. https://doi.org/10.3390/app7030263. 